Xenobiotica

the fate of foreign compounds in biological systems

ISSN: 0049-8254 (Print) 1366-5928 (Online) Journal homepage: http://www.tandfonline.com/loi/ixen20

\title{
Pregnancy affects the pharmacokinetics of sildenafil and its metabolite in the rabbit
}

\section{Francesca M. Russo, Paola Mian, Elke H. Krekels, Kristel Van Calsteren, Dick Tibboel, Jan Deprest \& Karel Allegaert}

To cite this article: Francesca M. Russo, Paola Mian, Elke H. Krekels, Kristel Van Calsteren, Dick Tibboel, Jan Deprest \& Karel Allegaert (2018): Pregnancy affects the pharmacokinetics of sildenafil and its metabolite in the rabbit, Xenobiotica, DOI: 10.1080/00498254.2017.1422217

To link to this article: https://doi.org/10.1080/00498254.2017.1422217

Accepted author version posted online: 26

Dec 2017.

Published online: 10 Jan 2018.

Submit your article to this journal \lceil

Џ Article views: 17

Q View related articles $₫$

View Crossmark data $₫$ 


\title{
Pregnancy affects the pharmacokinetics of sildenafil and its metabolite in the rabbit
}

\author{
Francesca M. Russo ${ }^{1,2} *$ (D) Paola Mian ${ }^{3} *$, Elke H. Krekels ${ }^{4}$, Kristel Van Calsteren ${ }^{1,2}$, Dick Tibboel ${ }^{3}$, Jan Deprest ${ }^{1,2,5}$ (D) \\ and Karel Allegaert ${ }^{1,3}$ \\ ${ }^{1}$ Cluster Woman and Child, Department of Development and Regeneration, Biomedical Sciences, KU Leuven, Leuven, Belgium, ${ }^{2}$ Department of \\ Obstetrics and Gynecology, University Hospitals Leuven, Leuven, Belgium, ${ }^{3}$ Intensive Care and Department of Paediatric Surgery, Erasmus MC \\ Sophia Children's Hospital, Rotterdam, The Netherlands, ${ }^{4}$ Division of Pharmacology, Leiden Academic Centre for Drug Research, Leiden University, \\ Leiden, The Netherlands, and ${ }^{5}$ Department of Obstetrics and Gynecology, Institute of Women's Health, University College London, London, UK
}

\section{Abstract}

1. There is growing interest in the use of sildenafil during pregnancy for various maternal and fetal conditions. This study aims to investigate the effect of pregnancy on the maternal pharmacokinetics (PK) of sildenafil and its main metabolite desmethylsildenafil in rabbits. Using NONMEM, population PK modeling was performed based on plasma samples from 31 rabbits of whom 15 were pregnant and 16 were not. All received a single subcutaneous sildenafil dose of $10 \mathrm{mg} / \mathrm{kg}$. One sample was obtained per rabbit at either 30, 60, 120, 360, 720 or 1320 min after sildenafil administration.

2. A two- and one-compartment PK-model best described the data for sildenafil and desmethylsildenafil, respectively. Compared to non-pregnant rabbits, the central and peripheral volume of distribution and inter-compartmental clearance of sildenafil were lower in pregnant rabbits [32.1 versus $12.2 \mathrm{~L}, 110$ versus $44.4 \mathrm{~L}$ and 25.5 versus $12.1 \mathrm{~L} / \mathrm{h}$; all $p<0.05]$. The formation clearance from sildenafil to desmethylsildenafil was also reduced during pregnancy [13.3 versus $7.8 \mathrm{~L} / \mathrm{h} ; p<0.05]$.

3. In contrast, the elimination clearance of desmethylsildenafil, was higher in pregnancy [73.5 versus 116. 9; $p<0.05$ ]. In rabbits, pregnancy impacts PK parameters of sildenafil and its metabolite, leading to an increased peak concentration and $24 \mathrm{~h}$ exposure for sildenafil and a decreased $24 \mathrm{~h}$ exposure for desmethylsildenafil.

\section{Keywords}

Pharmacokinetics, population pharmacokinetics modeling, pregnancy, sildenafil

\section{History}

Received 5 November 2017

Revised 23 December 2017

Accepted 24 December 2017

\section{Introduction}

Sildenafil is a selective phosphodiesterase 5 (PDE5) inhibitor registered for the treatment of erectile dysfunction (Nightingale, 1998) and pulmonary hypertension in adults (Galie et al., 2005). Apart from pulmonary arteries and the corpora cavernosum, PDE5 is abundantly expressed in various tissues, including the placenta and the fetus (Ribaudo et al., 2016). Hence, there has been a growing interest in the use of sildenafil during pregnancy for maternal and fetal conditions. In this unique setting, therapy involves at least two individuals, the pregnant mother and the fetus, both of them considered a special population. Therefore, demonstration of efficacy and tolerability in both subjects in relevant animal models is a critical step for clinical implementation.

\footnotetext{
*These authors contributed equally to this work.

Address for correspondence: Karel Allegaert, Cluster Woman and Child, Department of Development and Regeneration, Biomedical Sciences, KU Leuven, Leuven, Belgium. Tel: +32-16-343211. Fax: +32-16343209. E-mail: karel.allegaert@uzleuven.be
}

The rabbit is the third most widely used experimental mammal after mice and rats within the European Union (McArdle et al., 2009). As a model to study fetal and placental development, the rabbit presents several advantages over other species. Rabbits are widely available, have low housing needs, a timed gestation and a short reproductive cycle, with pregnancy lasting 31 days (Fischer et al., 2012). Similar to rodents, rabbits have large litters, allowing to test the effect of treatments on multiple fetuses at the same time. However, the relatively large size of rabbit fetuses compared with other laboratory animals makes functional assessments and noninvasive monitoring of fetal growth and wellbeing feasible (Hodges et al., 2013; Russo et al., 2016). The rabbit model also allows more detailed assessment of feto-maternal safety than rodents. Pregnancy-induced hemodynamic changes are comparable with those in women, i.e. with a steady increase in maternal blood pressure throughout gestation (Lee et al., 1982; McArdle et al., 2009). Furthermore, placental transfer in the latter half of pregnancy mirrors that in humans, since the rabbit mid- and endgestational placenta is functionally positioned between 
rodents (hemo-trichorial) and man (hemo-monochorial) (Enders and Blankenship, 1999; McArdle et al., 2009). This makes the rabbit a pertinent model for the study of transplacental therapies, including sildenafil.

It is known that pregnancy is associated with various physiological changes that may lead to significant variations in the pharmacokinetic (PK) profile of many drugs. However, in all clinical applications of sildenafil during pregnancy, the therapeutic dose has been extrapolated from studies in nonpregnant adults, as very limited information is available on the PK of sildenafil in pregnancy (Samangaya et al., 2009). Likewise, little information is present in the literature on the influence of pregnancy on drug metabolism in rabbits (Matar, 2013; Matar and Marafie, 2011). PK studies in the pregnant population are a critical first step before any study on transplacental therapy can be performed, both in translational and in clinical research. Such PK studies can be optimized by reducing the number of samples required, both for ethical and economic reasons. Population PK modeling is a useful tool to deal with sparse data sampling meeting the need to minimize the sample size (De Cock et al., 2011). Moreover, by using population PK-modeling sources of variability in PK can be identified.

The present study aims to investigate the effect of pregnancy on the PK of sildenafil and its main metabolite desmethylsildenafil in rabbits, by using population PK modeling.

\section{Materials and methods}

\section{In vivo study design}

This prospective study was approved by the Ethics Committee on Animal Experimentation of the Faculty of Medicine, KU Leuven, Leuven, Belgium (Project number: P014/2013). Hybrid Dendermonde-New Zealand white rabbits from a certified farm were used. Animals were housed in individual cages at normal room temperature on a 12/12-h light schedule with free access to food and water. They were treated in accordance with the Guide for the Care and Use of Laboratory Animals. Two experimental groups were included: nonpregnant $(N=16)$ and time mated pregnant rabbits at 24 days of gestation $(N=15)$. All animals received a single subcutaneous injection of $10 \mathrm{mg} / \mathrm{kg}$ sildenafil citrate (TEVA, Wilrijk, Belgium) diluted in saline solution at a concentration of $6 \mathrm{mg} / \mathrm{mL}$. Rabbits were randomized to obtain a single blood sample from the central ear artery at different scheduled time points either $30,60,120,360,720$ or $1320 \mathrm{~min}$ after sildenafil administration. Blood samples were collected in EDTA tubes and centrifuged at $3000 \mathrm{rpm}$ for $15 \mathrm{~min}$. The plasma was stored at $-80^{\circ} \mathrm{C}$ until analysis.

\section{Drug assay}

Plasma concentration of sildenafil and its active $N$-desmethyl metabolite desmethylsildenafil was measured using liquid chromatography-mass spectrometry (Vos et al., 2008) at the Department of Clinical Pharmacology and Pharmacy, VU University Medical Centre, Amsterdam, The Netherlands. The assay is linear over $1-600 \mathrm{ng} / \mathrm{mL}$ for sildenafil and $1-300 \mathrm{ng} / \mathrm{mL}$ for desmethylsildenafil, with the lower limit of the ranges representing the lower limits of quantification (LLOQ) of sildenafil and its metabolite. Its intraand inter-assay accuracies range from 92.8 to $103.2 \%$ and intra- and inter-assay imprecision does not exceed $15 \%$ (Vos et al., 2008).

\section{Population PK analysis and internal validation}

The population PK of sildenafil and desmethylsildenafil was characterized with non-linear mixed effects modeling using NONMEM version 7.2 software, subroutine ADVAN13, TOL9 (ICON Development Solutions, Ellicott City, MD). The first-order estimation method with the interactions option (FOCE-I) was used. Pirana (version 2.9.4.), R (version 3.3.0) and $\mathrm{PsN}^{\circledR}$ (version 4.4.8) were used to process runs, visualize data, and evaluate the model output. Concentrations of desmethylsildenafil were converted to sildenafil equivalents $(\mathrm{ng} / \mathrm{mL})$ based on molecular weights (molecular weight sildenafil: $474,5764 \mathrm{~g} / \mathrm{mol}$, molecular weight desmethylsildenafil: $460.55 \mathrm{~g} / \mathrm{mol}$ ) (PubChem).

The model building process was performed in a stepwise manner: (1) choice of the structural model, (2) choice of the statistical sub-model, (3) choice of the covariate model, and (4) model evaluation (Krekels et al., 2011). Discrimination between different models was made by the likelihood ratio test using the Objective Function Value (OFV) (i.e. $-2 \log$ likelihood). A decrease in OFV of 3.84 ( $p<0.05$ assuming a $\chi^{2}$ distribution) between nested models with one degree of freedom was considered statistically significant. The goodness-of-fit-plots, conditional weighted residuals versus population predicted concentrations, conditional weighted residuals versus time and observed versus population predicted concentrations were also evaluated. Finally, precision of the parameter estimates, parameter confidence intervals, and condition number were evaluated.

\section{Structural and statistical model}

One, two, and three compartment PK models for sildenafil and desmethylsildenafil concentrations were tested. In order to capture a possible delay in desmethylsildenafil formation, transit compartment models for the formation of the metabolite were also considered.

Because of the study design, some assumptions had to be made. The bioavailability after subcutaneous administration could not be identified without intravenous data and was therefore assumed to be $100 \%$. Consequently all model parameters were estimated relative to the fraction absorbed. The fraction of sildenafil that is converted to desmethylsildenafil in rabbits is unknown. However, conversion to desemethylsildenafil represents the main metabolic pathway both in humans and in rabbits (Walker et al., 1999). This fraction was therefore assumed to be $100 \%$ both in pregnant and non-pregnant rabbits; consequently volume of distribution and formation and elimination clearance parameters of the metabolite were estimated relative to the fraction metabolized (fm) (Qidwai et al., 2010). As absorption was near completion at the time of the first sample $(30 \mathrm{~min})$, the absorption rate constant (Ka) could not be estimated accurately and was therefore fixed to $25 \mathrm{~h}^{-1}$. At the end of model development, a sensitivity analysis was performed to estimate 
how this assumption affected the final estimates of the model parameters.

As only one sample per rabbit was obtained, intra- and inter-individual variability could not be identified separately. Therefore only residual variability was estimated, for this a proportional (Equation (1)), additive (Equation (2)) and a combined error model (combination of Equations (1) and (2)) were tested. This means for the $j$ th observed concentration $(\mathrm{Y})$ of the $i$ th individual the relation $\left(\mathrm{Y}_{i j}\right)$ :

$$
\begin{gathered}
Y_{i j}=C_{\text {pred, } i j} *\left(1+\varepsilon_{i j}\right) \\
Y_{i j}=C_{p r e d, i j} *\left(\varepsilon_{i j}\right)
\end{gathered}
$$

Where $C_{\text {pred,ij }}$ is the predicted concentrations and $\varepsilon_{i j}$ is a random value from a normal distribution with a mean of zero and an estimated variance of $\sigma^{2}$.

\section{Covariate model}

Both bodyweight and pregnancy were evaluated as potential covariates in the covariate analysis on all PK-parameters of both sildenafil and desmethylsildenafil.

Bodyweight was entered into the model using a linear or power equation (Equation (3)).

$$
P_{i}=\theta_{1} *\left(\frac{B W_{i}}{4.3}\right)^{X}+\theta_{2}
$$

In the equation $P_{i}$ represents individual parameter values, $\theta_{1}$ and $\theta_{2}$ represent the population parameter values for the covariate relationship and $\mathrm{BW}_{i}$ represents the covariate value bodyweight, which is normalized for the median bodyweight $(4.3 \mathrm{~kg})$ for the full population. $X$ is the exponent of the power function, which was fixed to 1 for a linear function or estimated for a power function. For pregnancy, the fractional change for the pregnant group was estimated compared to the non-pregnant group.

Potential covariates were separately entered into the model. When more than one significant covariate $(p<.05$, dOFV 3.84) was identified, the covariate causing the largest drop in OFV was retained in the model. Additional covariates had to further reduce this OFV to be retained. In addition, a reduction in the variance of the random effects was evaluated upon inclusion of the covariate on the parameters. The covariate model was further evaluated as discussed in the section "Population PK analysis and internal validation'.

\section{Internal validation}

The predictive performance of the model was evaluated with the normalized prediction distribution error (NPDE) method (Comets et al., 2008). In order to perform this analysis, the original dataset was simulated 1000 times. Each observed concentration was compared to the range of simulated concentrations for that observation using the NPDE package in R. A histogram of the NPDE distribution and scatterplots showing the NPDE versus time and versus predicted concentrations, stratified for sildenafil and desmethylsildenafil, were used to evaluate the final model.

\section{Simulations}

To examine the effect of pregnancy on the PK of sildenafil and its main metabolite, the final PK model was used to simulate concentration-time curves upon a $10 \mathrm{mg} / \mathrm{kg}$ subcutaneous injection in pregnant versus non-pregnant rabbits. The median bodyweight of the full study population was used to calculate the dose. Sildenafil and desmethylsildenafil concentrations were plotted versus time.

\section{Sample size calculation}

Since this is an observational pharmacokinetic study, and there is no information in the literature about sildenafil pharmacokinetics in rabbits after subcutaneous administration, no reliable sample size calculation is possible (Shannon et al., 1998). We assumed that a sample size of 3 animals per group for each time point is an ethical balance between the research question and the exposure of animals to the drug. Since the analysis of sildenafil levels showed low $(<20 \%)$ standard deviation within the groups, and since the population PK model can handle sparse sampling in a small population, we considered the sample size to be sufficient.

\section{Results}

Fifteen pregnant rabbits (median bodyweight [range] $4.6 \mathrm{~kg}$ [4.0-5.9]) and sixteen non-pregnant rabbits (3.9 kg [2.5-6.2]) were included in this study. Six rabbits (3 pregnant and 3 nonpregnant) were sampled at the same time point for $t=30,60$, 360,720 , and $1320 \mathrm{~min}$. An additional sample was collected from one non-pregnant rabbit at $120 \mathrm{~min}$ after injection. From each rabbit one sample was available for analysis. None of the samples for sildenafil or desmethylsildenafil were below the LLOQ.

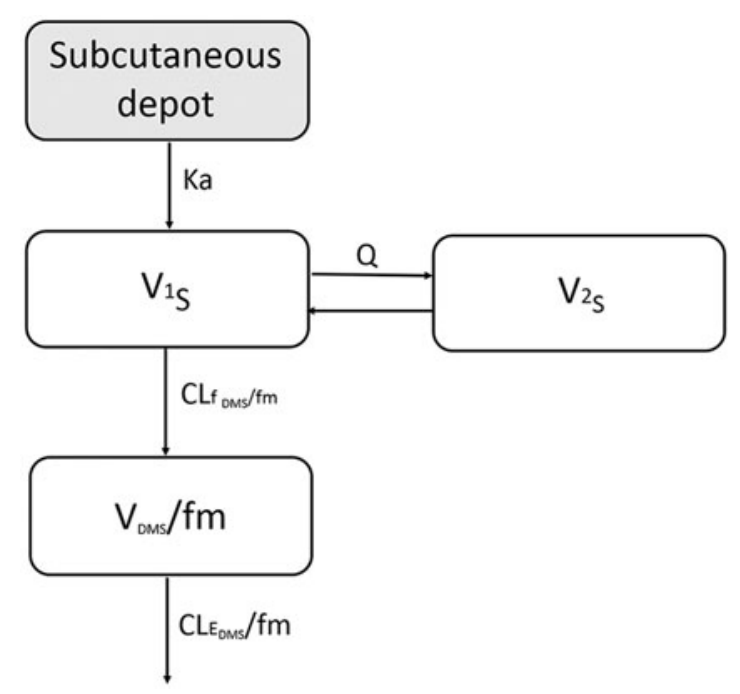

Figure 1. Schematic overview of the population PK model of sildenafil (S) and desmethylsildenafil (DMS). Ka: absorption rate constant of sildenafil from the subcutaneous depot compartment, $\mathrm{V} 1_{\mathrm{S}}$ : central volume of distribution for sildenafil, $\mathrm{V} 2 \mathrm{~s}$ : peripheral volume of distribution for sildenafil, Q: inter-compartmental clearance for sildenafil, $\mathrm{CLf}_{\mathrm{DMS} / \mathrm{fm}}$ : formation clearance of sildenafil to desmethylsildenafil, $\mathrm{VD}_{\mathrm{MS} / \mathrm{fm}}$ : volume of distribution of desmethylsildenafil, $\mathrm{CLe}_{\mathrm{DMS} / \mathrm{fm}}$ : elimination clearance of desmethylsildenafil. $\mathrm{fm}=$ fraction metabolized to desmethylsildenafil. All model parameters are relative to the subcutaneous bioavailability. 
Table 1. PK parameters of the final population PK model for sildenafil and desmethylsildenafil in pregnant and non-pregnant rabbits.

\begin{tabular}{|c|c|c|}
\hline \multicolumn{3}{|c|}{ Final model } \\
\hline Parameter & Non-pregnant rabbits (RSE\%) & Pregnant rabbits (RSE\%) \\
\hline \multicolumn{3}{|l|}{ Sildenafil } \\
\hline$C L f_{D M S} / f m(\mathrm{~L} / \mathrm{h})$ & $13.3(5.7)$ & $13.3 * 0.589(8)=7.8$ \\
\hline$V 1_{S}(\mathrm{~L})$ & $32.1(11.9)$ & $32.1 * 0.38(27.6)=12.2$ \\
\hline$V 2_{S}(\mathrm{~L})$ & $110.0(16.2)$ & $110.0 * 0.404(18.7)=44.4$ \\
\hline$Q(\mathrm{~L} / \mathrm{h})$ & $25.5(15.6)$ & $25.5^{*} 0.471(19.8)=12.0$ \\
\hline$K a\left(\mathrm{~h}^{-1}\right)$ & \multicolumn{2}{|c|}{25 FIX } \\
\hline \multicolumn{3}{|l|}{ Desmethylsildenafil } \\
\hline$V_{D M S} / f m(\mathrm{~L})$ & \multicolumn{2}{|c|}{$226.0(12.7)$} \\
\hline$C l e_{D M S} / f m(\mathrm{~L} / \mathrm{h})$ & $73.5(10.5)$ & $73.5 * 1.59(16.9)=116.9$ \\
\hline \multicolumn{3}{|l|}{ Residual variability $\left[\sigma^{2}\right]$ (RSE\%) } \\
\hline Proportional error sildenafil & \multicolumn{2}{|c|}{$0.0435(22)$} \\
\hline Proportional error desmethylsildenafil & \multicolumn{2}{|c|}{$0.15(24)$} \\
\hline
\end{tabular}

The parameter values in pregnant rabbits are presented as the parameter values multiplied by the estimated fraction. RSE: relative standard error, $K a$ : absorption rate constant of sildenafil, $V 1_{S}$ : central volume of distribution for sildenafil, $V 2_{S}$ : peripheral volume of distribution for sildenafil, $Q$ : intercompartmental clearance of sildenafil, $C L f_{D M S} / f m$ : formation clearance from sildenafil to desmethylsildenafil, $V_{D M S} / f m$ : central volume of distribution of desmethylsildenafil, $\mathrm{Cl}_{D M S} / \mathrm{fm}$ : elimination clearance of desmethylsildenafil, fm: fraction metabolized to desmethylsildenafil. All parameters are relative to the subcutaneous bioavailability.

\section{Structural and statistical model}

A two-compartment model for sildenafil and a one-compartment model for desmethylsildenafil best fitted the data (Figure 1). Proportional error models best described residual variability for the sildenafil and desmethylsildenafil concentrations.

\section{Covariate model}

Parameter estimates of the final model in pregnant and nonpregnant rabbits are provided in Table 1 . Pregnancy was a significant covariate for the central $\left(\mathrm{V} 1_{\mathrm{S}}\right)$ and peripheral $\left(\mathrm{V} 2_{\mathrm{S}}\right)$ volume of distribution of sildenafil, resulting in a lower $\mathrm{V} 1_{\mathrm{S}}(-62 \%)$ and $\mathrm{V} 2_{\mathrm{S}}(-60 \%)$ in pregnant rabbits compared to non-pregnant rabbits. In addition, the inter-compartmental clearance of sildenafil $(Q)$ was significantly lower in pregnancy $(-53 \%)$ The formation clearance from sildenafil to desmethylsildenafil $\left(\mathrm{CLf}_{\mathrm{DMS}} / \mathrm{fm}\right)$ was lower in pregnant as compared to non-pregnant rabbits $(-41 \%)$ while the elimination clearance of desmethylsildenafil $\left(\mathrm{CLE}_{\mathrm{DMS}} / \mathrm{fm}\right)$ was higher in pregnant animals $(+40 \%)$. After inclusion of pregnancy in the model, bodyweight did not significantly improve the model further. The sensitivity analysis performed for the fixed absorption rate constant showed no effect on the final model parameters when Ka from 2.5 to $250 \mathrm{~h}^{-1}$ was tested, as the differences between the estimated values were less than $10 \%$.

Goodness-of-fit plots of the final model is shown in Figure 2. The population predicted and observed concentrations for both pregnant and non-pregnant rabbits were evenly distributed around the line of unity, indicating that the model describes the concentrations accurately. The conditional weighted residuals over time and over the concentration range were also unbiased, suggesting there is no time or concentration related bias in the model predictions.

\section{Internal validation}

The NPDEs for sildenafil and desmethylsildenafil are depicted in Figure 3. The histogram followed the normal distribution. No trend was seen in the NPDE versus time and the NPDE versus predicted concentrations, suggesting unbiased model predictions of sildenafil and desmethylsildenafil concentrations over time and over the concentration range.

\section{Simulations}

Figure 4 shows population-predicted sildenafil and desmethylsildenafil concentrations after a subcutaneous injection of $10 \mathrm{mg} / \mathrm{kg}$ sildenafil in pregnant and non-pregnant rabbits of the same bodyweight. As a result of the lower central and peripheral volume of distribution of sildenafil, the maximum concentration $(\mathrm{Cmax})$ for sildenafil is higher in pregnant rabbits compared to non-pregnant rabbits (Figure 4(A)). Furthermore, as a result of a lower formation clearance from sildenafil to desmethylsildenafil, the area under the curve (AUC) from 0 to $24 \mathrm{~h}$ of sildenafil is higher in pregnant rabbits. However, when considering the $\mathrm{AUC}_{0-\infty}$, the difference between pregnant and non-pregnant rabbits diminishes due to differences in peripheral distribution of the drug. In addition, an effect of pregnancy on the elimination clearance of desmethylsildenafil resulted in a lower $\mathrm{AUC}_{0-24 \mathrm{~h}}$ in pregnant rabbits (Figure 4(B)).

\section{Discussion}

We investigated the effect of pregnancy on the PK of sildenafil and its main metabolite in rabbits as a representative preclinical model. We observed a significant $(p<0.05)$ lower volume of distribution of sildenafil in pregnant rabbits compared to non-pregnant rabbits, resulting in a higher predicted maximum concentration (Figure 4(A)). 
(A)

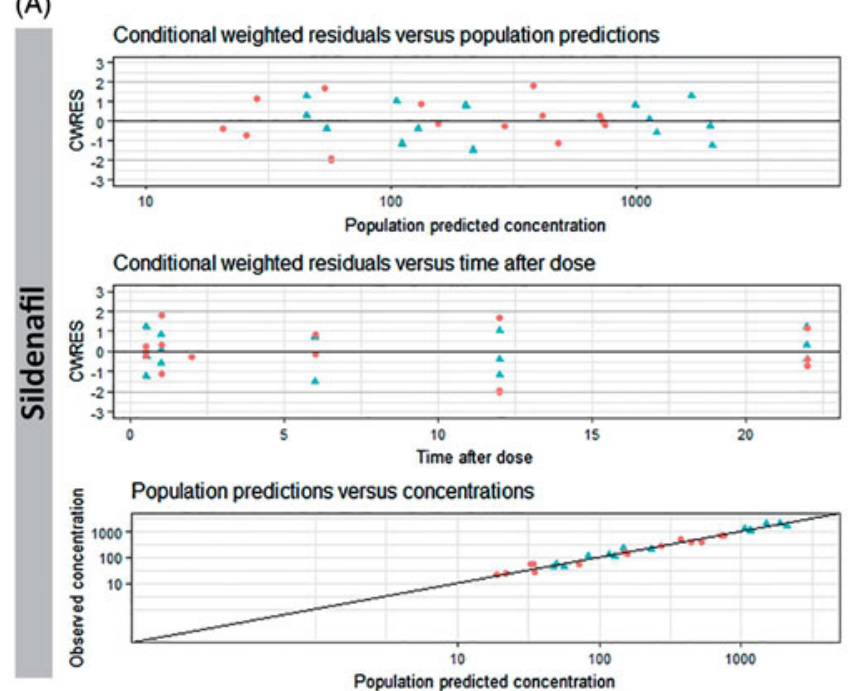

(B)

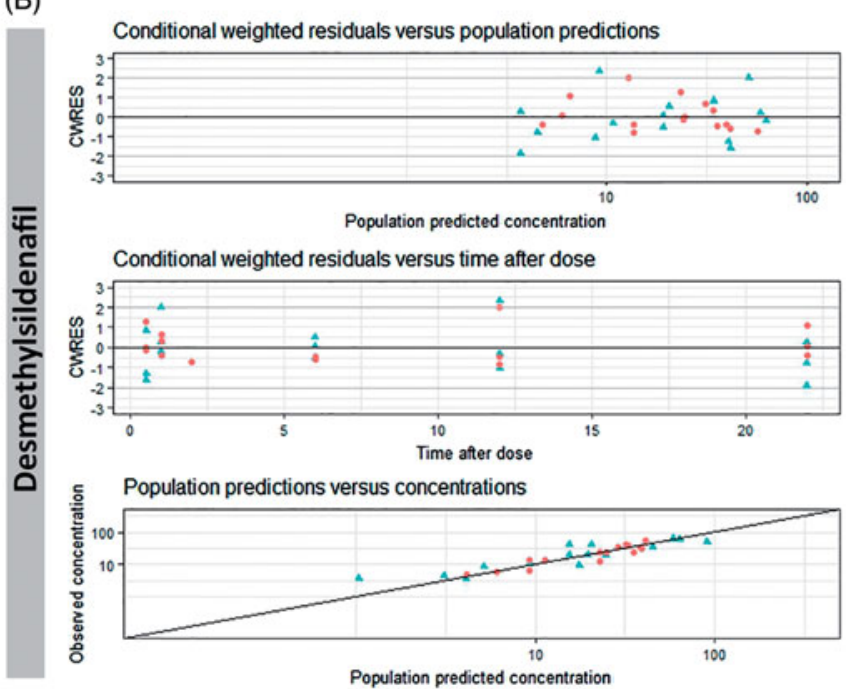

Figure 2. Goodness-of-fit plots of pregnant (blue, triangle) and non-pregnant (red, circle) rabbits for sildenafil (panel A) and desmethylsildenafil (panel B). The $y$ - and $x$ - axis of the population predictions versus concentrations and the $x$-axis of conditional weighted residuals (CWRES) versus population predicted concentration are on a log-scale.

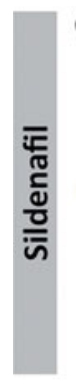

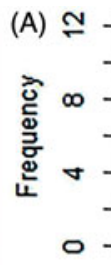

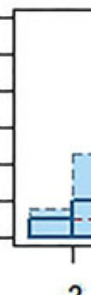

$-2$

Sample quantiles (npde)

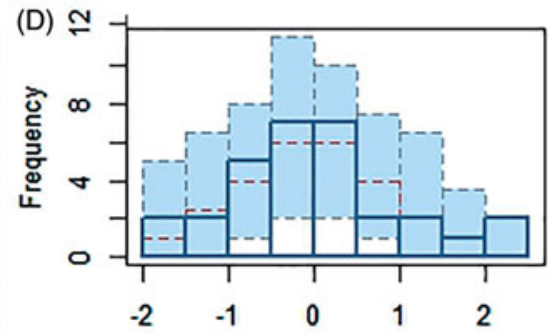

Sample quantiles (npde)

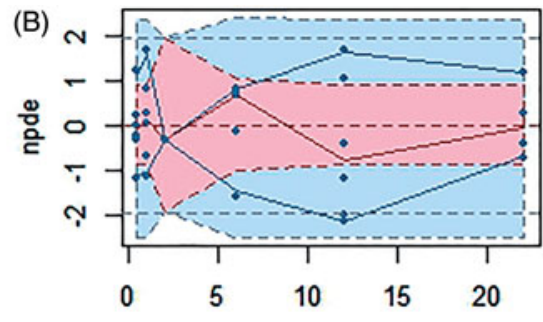

TIME (min)

(E)

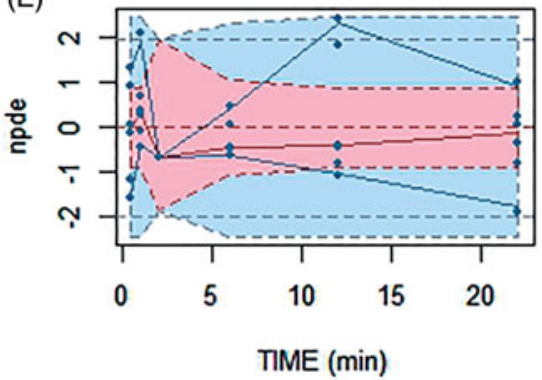

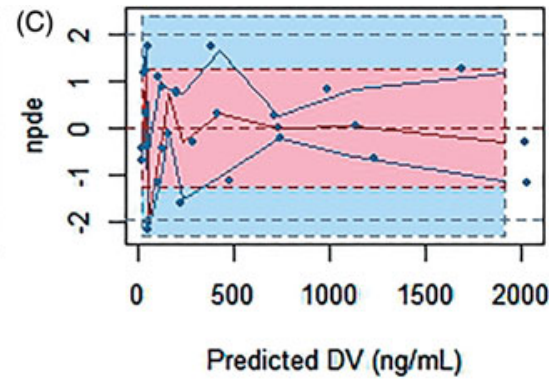

(F)

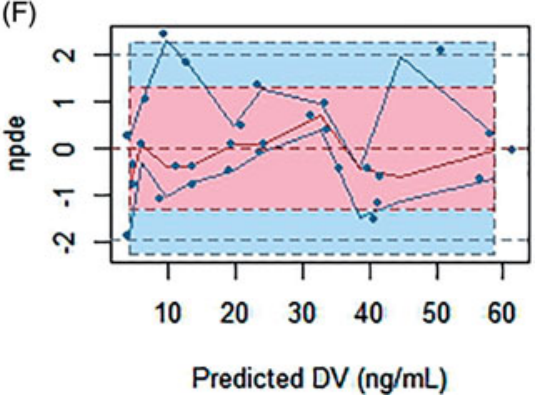

Figure 3. Normalized prediction distribution error (NPDE) of the final model for sildenafil (top panels, A-C) and desmethylsildenafil (bottom panels, D-F) in pregnant and non-pregnant rabbits. The histograms show the NPDE distribution for sildenafil (A) and desmethylsildenafil (D). The distribution of the NPDE versus time after dose (B, E) and NPDE versus the concentrations $(\mathrm{C}, \mathrm{F})$ are also illustrated.

Furthermore, as a result of lower formation clearance $(p<0.05)$ from sildenafil to desmethylsildenafil, the $\mathrm{AUC}_{0-}$ $24 \mathrm{~h}$ of sildenafil was higher in pregnant rabbits. Finally, pregnancy was associated with an increase in elimination clearance $(p<0.05)$ of desmethylsildenafil, resulting in a lower $\mathrm{AUC}_{0-24 \mathrm{~h}}$ for the metabolite (Figure 4(B)).

These results are counterintuitive. Sildenafil is primarily metabolized by the CYP3A4 isoenzyme, and to a lesser extent CYP2C9 (Muirhead et al., 2002; Walker et al., 1999). In humans, activity of these cytochrome isoforms is induced during pregnancy, which contributes to the increased clearance of many drugs (Feghali et al., 2015; Hebert et al., 2008).
PK studies in rabbits on drugs metabolized by the same isoforms have shown a similar increase in clearance (Matar, 2013; Matar and Marafie, 2011). This is in contrast with what we observed for sildenafil. Furthermore, sildenafil is highly bound to plasma protein both in humans and rabbits (Walker et al., 1999). An increase in the elimination clearance of sildenafil would then also be expected as a result of the decrease in plasma proteins concentrations associated to pregnancy (Cheung et al., 1989). Finally, an increase in plasma volume caused by pregnancy is expected (Qasqas et al., 2004). For sildenafil we observed a decrease in volume of distribution, while no effect of pregnancy has been 
Figure 4. Population predicted sildenafil concentrations (A) and desmethylsildenafil concentrations (B) over time in pregnant (blue, dotted line) and non-pregnant rabbits (red, solid line) weighing $4.3 \mathrm{~kg}$ after $10 \mathrm{mg} / \mathrm{kg}$ subcutaneous sildenafil.
(A)

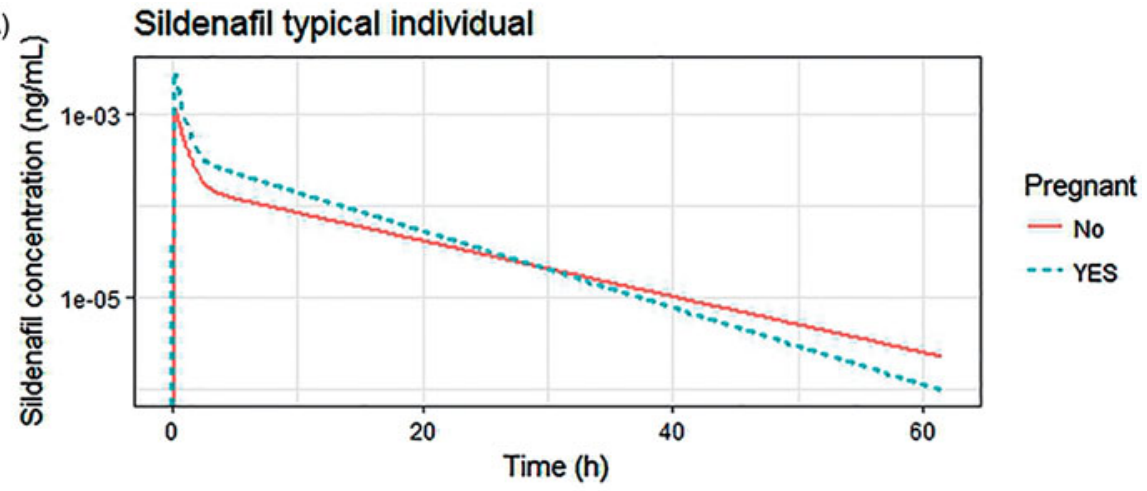

(B)

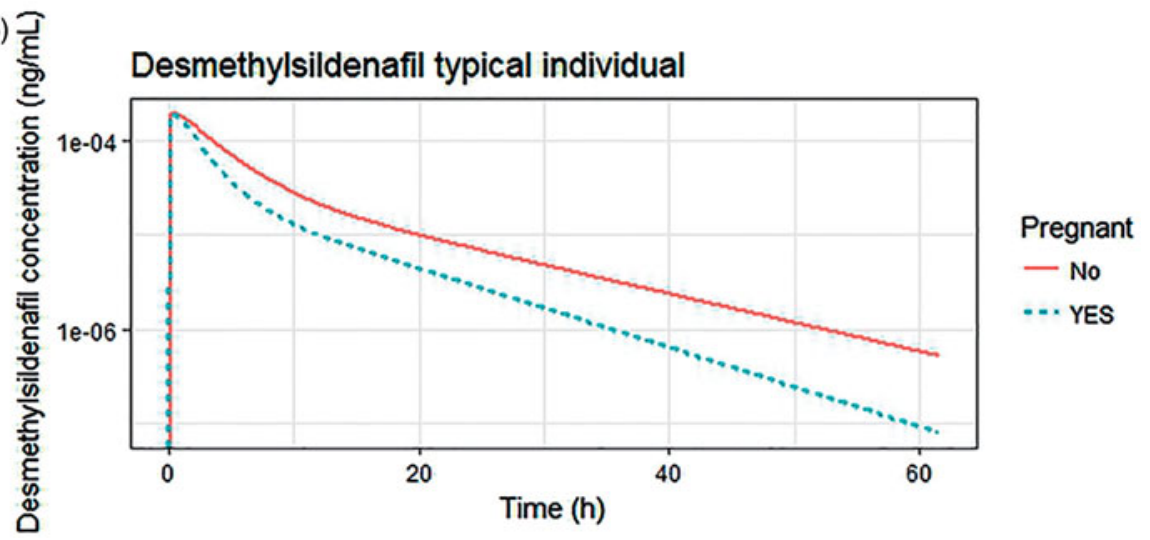

observed on the volume of distribution of its metabolite. The elimination clearance of sildenafil in desmethylsildenafil is increased in pregnancy.

As no data after intravenous administration were available, it was assumed that $100 \%$ of the administered subcutaneous dose was absorbed. Furthermore, since we had no urine samples, absolute sildenafil amounts being eliminated through the different routes could not be established. Walker et al. reported that sildenafil is mostly metabolized to $N$-desmethylsildenafil and in a much lesser extent through other metabolic pathways in the non-pregnant rabbit (Walker et al., 1999). Therefore, and as only $N$-desmethylsildenafil as a metabolite was measured, the assumption of $100 \%$ conversion was made. Consequently, if the fraction of formation to other metabolites and absorption is known in both pregnant and non-pregnant rabbits all the PK parameters should decrease proportionally and if the fraction metabolized to desmethylfildenafil would be less than $100 \%$, all parameters related to the metabolite would also decrease proportionally.

This is the first study comparing sildenafil PK in pregnant and non-pregnant subjects, using a rabbit model. The counterintuitive differences in PK parameters suggest that additional, still unexplored, mechanisms may contribute to the effect of pregnancy on sildenafil metabolism and distribution. This is of interest for future animal research, both on sildenafil and on other compounds with a therapeutic indication during pregnancy. This is of relevance, since several new indications for sildenafil in pregnancy are claimed and are investigated in animal studies. To guarantee the translational impact of such studies, demonstration that the drug is administered at the target therapeutic dose in the study population - be it animal experimental or human-is mandatory. We chose for limited sampling per animal, to reduce individual burden. Population modeling can still deal with this data and the improved statistical power compared to more traditional data analysis techniques.

Furthermore, given the minimal impact on animal welfare, a single drug administration followed by a single blood sample can potentially be performed in animals already included for other experimental purposes. As a result, such PK studies might be accomplished without additional animals, in agreement with the 3R's rules on Humane Experimental Techniques (Russell, 1995).

The results from this study also underline the need for PK studies in pregnant patients using sildenafil. Sildenafil treatment during pregnancy is becoming more common in the clinical setting. Several case reports have been published on the chronic use of sildenafil in pregnant patients with pulmonary hypertension (Lacassie et al., 2004; Molelekwa et al., 2005; Streit et al., 2009). Sildenafil has also been evaluated as a treatment of placentation disorders, such as preeclampsia (Samangaya et al., 2009) and intrauterine growth restriction (von Dadelszen et al., 2011), and in pregnancies complicated by oligohydramnios (Maher et al., 2017). Currently, three phase III trials on sildenafil treatment during pregnancy are ongoing, two in cases with intrauterine growth restriction (Ganzevoort et al., 2014), and one in normal pregnancies to reduce the risk of fetal distress during labor (Dunn et al., 2016). Despites these practices, we are unaware of a sildenafil PK study specifically in pregnant patients. Only one study provides limited information on maximum and mean sildenafil plasma concentrations after 
administration at different doses (Samangaya et al., 2009). However, this study was conducted in patients having preeclampsia, which is associated with changes in body composition, liver and kidney function that can profoundly influence drug metabolism (Ganzevoort et al., 2004; Jeyabalan and Conrad, 2007).

The pregnancy-related alterations in the PK profiles of sildenafil and its metabolite as shown in our study, demonstrates that extrapolation from non-pregnant to pregnant subjects might be hazardous, or should at least be validated in pregnant women.

We acknowledge the limitations of this study. First, sildenafil was administered in pregnant rabbits at 24 days of gestation, corresponding to the late second/early third trimester. This gestational age was selected because it corresponds to the gestational period in which sildenafil is indicated for most of the aforementioned pregnancy complications in the clinical setting. Also, the physiological alterations related to pregnancy are expected to be most profound in the second half of the pregnancy (Matar and Marafie, 2011). Due to this study design, pregnancy could only be studied as a dichotomous covariate, while changes in plasma volume, blood perfusion, kidney and liver function are gestational age-specific (Feghali et al., 2015). Potential differences in sildenafil PK across gestation were therefore not investigated. Secondly, the preferred route of drug administration in a clinical setting is oral, while we used subcutaneous administration. We assessed different routes and regimens in pilot rabbit studies (data not published). Oral administration could not guarantee a controlled intake of the right dose in the rabbits, unless done via gavage. However, most of the animal studies on sildenafil in pregnancy require chronic daily administration, often together with fetal manipulations necessary for outcome assessment. Repeated gavages in this setting would then induce excessive stress in the animal, increasing the risk of fetal loss and maternal discomfort. On the contrary, daily subcutaneous injections at a dose of $10 \mathrm{mg} / \mathrm{kg}$ led to sildenafil plasma levels within the therapeutic range (Russo et al., 2016) with minimal stress for the animal. However, the effect of pregnancy on gastrointestinal absorption and pre-systemic metabolism (e.g. enterocyte), as well as the influence of food intake on PK parameters following oral administration (Nichols et al., 2002) have to be considered when translating the results of this study to the human setting. Finally, the maternal PK data are only the first, but crucial step to further investigate transplacental transfer and fetal PK. This is especially of relevance when the fetus represents the target compartment since there is recent preclinical evidence that the drug may be used in women carrying fetuses with congenital diaphragmatic hernia (CDH) to prevent the occurrence of pulmonary hypertension after birth (Luong et al., 2011; Mous et al., 2016; Russo et al., 2016).

In conclusion, the present study shows that the PK profile of sildenafil and its metabolite in rabbits is changed during the second half of gestation, leading to higher predicted peak concentration and 24-h exposure for sildenafil and lower 24-h exposure for its metabolite in pregnant rabbits. This information might be of relevance for future animal studies as well as for the design of PK studies in humans.

\section{Declaration of interest}

No potential conflict of interest was reported by the authors.

Karel Allegaert has been supported by the Fund for Scientific Research, Flanders (fundamental clinical investigatorship $1800214 \mathrm{~N}$ ), and his research activities are further facilitated by the agency for innovation by Science and Technology in Flanders (IWT) through the SAFEPEDRUG project (IWT/SBO 130033). The Leuven program on medical therapy of $\mathrm{CDH}$ is funded by the Brianna Memorial Research Fund and the KU Leuven (C3/17/054, and C3/15/353). JDP is supported by the Great Ormond Street Hospital Charity Fund.

\section{ORCID}

Francesca M. Russo (D) http://orcid.org/0000-0002-5029-7899 Jan Deprest (D) http://orcid.org/0000-0002-4920-945X

Karel Allegaert (D) http://orcid.org/0000-0001-9921-5105

\section{References}

Cheung CK, Lao T, Swaminathan R. (1989). Urinary excretion of some proteins and enzymes during normal pregnancy. Clin Chem 35: 1978-80.

Comets E, Brendel K, Mentre F. (2008). Computing normalised prediction distribution errors to evaluate nonlinear mixed-effect models: the NPDE add-on package for R. Comput Methods Programs Biomed 90:154-66.

De Cock RF, Piana C, Krekels EH, et al. (2011). The role of population PK-PD modelling in paediatric clinical research. Eur J Clin Pharmacol 67(Suppl 1):5-16.

Dunn L, Flenady V, Kumar S. (2016). Reducing the risk of fetal distress with sildenafil study (RIDSTRESS): a double-blind randomised control trial. J Transl Med 14:15.

Enders AC, Blankenship TN. (1999). Comparative placental structure. Adv Drug Deliv Rev 38:3-15.

Feghali M, Venkataramanan R, Caritis S. (2015). Pharmacokinetics of drugs in pregnancy. Semin Perinatol 39:512-19.

Fischer B, Chavatte-Palmer P, Viebahn C, et al. (2012). Rabbit as a reproductive model for human health. Reproduction 144:1-10.

Galie N, Ghofrani HA, Torbicki A, et al. (2005). Sildenafil citrate therapy for pulmonary arterial hypertension. N Engl J Med 353:2148-57.

Ganzevoort W, Alfirevic Z, Von Dadelszen P, et al. (2014). STRIDER: sildenafil therapy in dismal prognosis early-onset intrauterine growth restriction-a protocol for a systematic review with individual participant data and aggregate data meta-analysis and trial sequential analysis. Syst Rev 3:23.

Ganzevoort W, Rep A, Bonsel GJ, et al. (2004). Plasma volume and blood pressure regulation in hypertensive pregnancy. J Hypertens 22: 1235-42.

Hebert MF, Easterling TR, Kirby B, et al. (2008). Effects of pregnancy on CYP3A and P-glycoprotein activities as measured by disposition of midazolam and digoxin: a University of Washington specialized center of research study. Clin Pharmacol Ther 84:248-53.

Hodges R, Endo M, La Gerche A, et al. (2013). Fetal echocardiography and pulsed-wave Doppler ultrasound in a rabbit model of intrauterine growth restriction. J Vis Exp 2013;76. doi: 10.3791/50392.

Jeyabalan A, Conrad KP. (2007). Renal function during normal pregnancy and preeclampsia. Front Biosci 12:2425-37.

Krekels EH, Van Hasselt JG, Tibboel D, et al. (2011). Systematic evaluation of the descriptive and predictive performance of paediatric morphine population models. Pharm Res 28:797-811.

Lacassie HJ, Germain AM, Valdes G, et al. (2004). Management of Eisenmenger syndrome in pregnancy with sildenafil and L-arginine. Obstet Gynecol 103:1118-20.

Lee MI, Oakes GK, Lam R, Hobel CJ. (1982). The rabbit: a suitable model for investigation of vascular responsiveness during pregnancy. Clin Exp Hypertens B 1:429-39.

Luong C, Rey-Perra J, Vadivel A, et al. (2011). Antenatal sildenafil treatment attenuates pulmonary hypertension in experimental congenital diaphragmatic hernia. Circulation 123:2120-31. 


\section{F. M. Russo et al.}

Maher MA, Sayyed TM, Elkhouly N. (2017). Sildenafil citrate therapy for oligohydramnios: a randomized controlled trial. Obstet Gynecol 129:615-20.

Matar KM. (2013). Impact of pregnancy on zonisamide pharmacokinetics in rabbits. Biomed Res Int 2013:140327.

Matar KM, Marafie NA. (2011). Effect of pregnancy on topiramate pharmacokinetics in rabbits. Xenobiotica 41:416-21.

Mcardle AM, Denton KM, Maduwegedera D, et al. (2009). Ontogeny of placental structural development and expression of the reninangiotensin system and 11beta-HSD2 genes in the rabbit. Placenta 30: $590-8$.

Molelekwa V, Akhter P, Mckenna P, et al. (2005). Eisenmenger's syndrome in a 27 week pregnancy-management with bosentan and sildenafil. Ir Med J 98:87-8.

Mous DS, Kool HM, Buscop-Van Kempen MJ, et al. (2016). Clinically relevant timing of antenatal sildenafil treatment reduces pulmonary vascular remodeling in congenital diaphragmatic hernia. Am J Physiol Lung Cell Mol Physiol 311:L734-1742.

Muirhead GJ, Rance DJ, Walker DK, Wastall P. (2002). Comparative human pharmacokinetics and metabolism of single-dose oral and intravenous sildenafil citrate. Br J Clin Pharmacol 53:13s-20s.

Nichols DJ, Muirhead GJ, Harness JA. (2002). Pharmacokinetics of sildenafil after single oral doses in healthy male subjects: absolute bioavailability, food effects and dose proportionality. $\mathrm{Br} \mathrm{J}$ Clin Pharmacol 53(Suppl 1):5s-12s.

Nightingale SL. (1998). From the food and drug administration. JAMA 279:1684.

Pubchem, desmethylsildenafil [online]. Available from: https://pubchem.ncbi.nlm.nih.gov/compound/6426858 [Last accessed 23 December 2017].

Pubchem, Sildenafil [online]. Available from: https://pubchem.ncbi.nlm.nih.gov/compound/sildenafil [Last accessed 23 December 2017].

Qasqas SA, Mcpherson C, Frishman WH, Elkayam U. (2004). Cardiovascular pharmacotherapeutic considerations during pregnancy and lactation. Cardiol Rev 12:240-61.
Qidwai K, Pearson DM, Patel GS, et al. (2010). Deletions of Xp provide evidence for the role of holocytochrome $\mathrm{C}$-type synthase (HCCS) in congenital diaphragmatic hernia. Am J Med Genet A 152a: 1588-90.

Ribaudo G, Pagano MA, Bova S, Zagotto G. (2016). New therapeutic applications of phosphodiesterase 5 inhibitors (PDE5-Is). Curr Med Chem 23:1239-49.

Russell WM. (1995). The development of the three Rs concept. Altern Lab Anim 23:298-304.

Russo FM, Toelen J, Eastwood MP, et al. (2016). Transplacental sildenafil rescues lung abnormalities in the rabbit model of diaphragmatic hernia. Thorax 71:517-25.

Samangaya RA, Mires G, Shennan A, et al. (2009). A randomised, double-blinded, placebo-controlled study of the phosphodiesterase type 5 inhibitor sildenafil for the treatment of preeclampsia. Hypertens Pregnancy 28:369-82.

Shannon C, Jauniaux E, Gulbis B, et al. (1998). Placental transfer of fentanyl in early human pregnancy. Hum Reprod $13: 2317-20$.

Streit M, Speich R, Fischler M, Ulrich S. (2009). Successful pregnancy in pulmonary arterial hypertension associated with systemic lupus erythematosus: a case report. J Med Case Rep 3: 7255 .

Von Dadelszen P, Dwinnell S, Magee LA, et al. (2011). Sildenafil citrate therapy for severe early-onset intrauterine growth restriction. BJOG 118:624-8.

Vos RM, Chahbouni A, Sinjewel A, Swart EL. (2008). Quantitative analysis of sildenafil and desmethylsildenafil in human serum by liquid chromatography-mass spectrometry with minimal sample pretreatment. J Chromatogr B Analyt Technol Biomed Life Sci 876: 283-7.

Walker DK, Ackland MJ, James GC, et al. (1999). Pharmacokinetics and metabolism of sildenafil in mouse, rat, rabbit, dog and man. Xenobiotica 29:297-310. 